\title{
Explicit Shifted Second-kind Chebyshev Spectral Treatment for Fractional Riccati Differential Equation
}

\author{
W. M. Abd-Elhameed ${ }^{1,2,}$, and Y. H. Youssri ${ }^{2}$
}

\begin{abstract}
This paper is confined to analyzing and implementing new spectral solutions of the fractional Riccati differential equation based on the application of the spectral tau method. A new explicit formula for approximating the fractional derivatives of shifted Chebyshev polynomials of the second kind in terms of their original polynomials is established. This formula is expressed in terms of a certain terminating hypergeometric function of the type ${ }_{4} F_{3}(1)$. This hypergeometric function is reduced in case of the integer case into a certain terminating hypergeometric function of the type ${ }_{3} F_{2}(1)$ which can be summed with the aid of Watson's identity. Six illustrative examples are presented to ensure the applicability and accuracy of the proposed algorithm.
\end{abstract}

Keywords: Chebyshev polynomials of the second kind, spectral methods, linearization formula, hypergeometric functions.

\section{Introduction}

The class of Jacobi polynomials has occupied a great deal of attention from both theoretical and practical points of view. Six celebrated special polynomials of Jacobi polynomials were extensively used for various purposes. Four kinds of Chebyshev polynomials are special kinds of Jacobi polynomials. The first and second kinds are ultraspherical polynomials, while the third and fourth kinds are not. For some theoretical and practical applications of the four kinds, one can be referred to Doha et al. [Doha, Abd-Elhameed and Youssri (2013); Abd-Elhameed, Doha, Youssri et al. (2016); Doha, Abd-Elhameed and Bassuony (2015)]. Masjed-Jamei [Masjed-Jamei (2006)] has introduced two new kinds of Chebyshev polynomials, and he called them fifth- and sixth- kinds of Chebyshev polynomials. The reason for calling them Chebyshev polynomials was due to their trigonometric representations. Some numerical algorithms for solving some types of fractional differential equations were developed based on employing the fifth- and sixth-kinds, see Abd-Elhameed et al. [Abd-Elhameed and Youssri $(2018,2019)]$.

\footnotetext{
${ }^{1}$ Department of Mathematics, Faculty of Science, University of Jeddah, Jeddah, Saudi Arabia.

${ }^{2}$ Department of Mathematics, Faculty of Science, Cairo University, Giza, 12613, Egypt.

*Corresponding Author: W. M. Abd-Elhameed. Email: waleed@ sci.cu.edu.eg.
} 
The branch of fractional calculus is an old branch but its importance has increased in the recent decades due to its broad applications in almost all disciplines of applied sciences. For example, they arise in the branches of mathematics, engineering, physics, fluid mechanics, electrochemistry, optics, and medicine. For some applications of the fractional calculus, one can be refereed to sun et al. [Sun, Zhang, Baleanu et al. (2018); Anastassiou and Argyros (2016); Rudolf (2000)]. Several contributions were performed for investigating fractional initial and boundary value problems. In this regard, a variety of numerical techniques were utilized to solve different fractional differential equations. Some of the used methods are: the finite difference method [Meerschaert and Tadjeran (2006)], Adomian's decomposition method [Daftardar-Gejji and Jafari (2007)], wavelets methods [Abd-Elhameed and Youssri (2015)], and operational matrix methods [Abd-Elhameed and Youssri (2016a,b)]. Some other recent methods can be found in Jena et al. [Jena, Chakraverty and Baleanu (2019); Jena, Chakraverty and Jena (2019); Alquran and Jaradat (2018); Ali, Alquran and Jaradat (2019)].

Riccati differential equation is of great importance in a variety of disciplines of applied science, see for instance Einicke et al. [Einicke, White and Bitmead (2003)]. The fractional Riccati differential equation has been investigated by proposing a large number of analytical and numerical algorithms. Some of the analytic used methods are: homotopy perturbation method [Khan, Ara and Jamil (2011)], modified homotopy perturbation method [Odibat and Momani (2008)], the Legendre wavelet operational matrix method [Balaji (2015)], Taylor collocation method [Öztürk, Anapalı, Gülsu et al. (2013)] and Jacobi collocation method [Singh and Srivastava (2019)]. For some other studies concerning these kinds of equations, one can be refereed to khan et al. [Khan, Ara and Khan (2013); Salehi and Darvishi (2016); Khader (2013)].

The problem of representing the high-order integer derivatives of different orthogonal polynomials in terms of their original polynomials is a very important problem in spectral and pseudospectral methods, since these explicit formulas if available serve in obtaining various spectral solutions using the different versions of spectral methods. Such derivatives for Jacobi polynomials can be found in Doha [Doha (2002)], and for Chebyshev polynomials of the third and fourth kinds can be found in Doha et al. [Doha, Abd-Elhameed and Bassuony (2015)].

The approach of employing fractional operational matrices of derivatives was successfully followed by a large number of authors. The use of these operational matrices along with utilizing suitable spectral methods enable one to obtain semi analytic solutions for a variety of fractional differential equations (see, for example Abd-Elhameed et al. [Abd-Elhameed and Youssri $(2016 a, b)])$. In the current paper, instead of employing the operational matrices of derivatives, we propose a new and different technique which is built on deriving explicit formula for the fractional derivatives of the shifted Chebyshev of the second kind, and after that utilize the tau spectral method for proposing semi analytic solution of fractional Riccati differential equation. To the best of our knowledge, this approach is new and differs from the other approaches used for handling fractional differential equations. We do believe that 
this new approach can be used for treating other types of fractional differential equations.

The structure of the paper is as follows. In Section 2, some preliminaries of the fractional calculus theory are presented and also some properties of Chebyshev polynomials of the second kind are exhibited. In Section 3, a new formula expressing approximately the fractional derivatives of the shifted Chebyshev polynomials of the second kind is established. Moreover, we show that the well-known formula of the integer derivatives can be extracted from this formula. In Section 4, we analyze and implement a numerical method based on utilizing the spectral tau method for solving the fractional Riccati differential equation. Section 5 is devoted to the investigation of the convergence and error analysis. In Section 6, some illustrative numerical examples are presented aiming to validate the accuracy and the efficiency of the proposed algorithm. Finally, some concluding remarks are given in Section 7.

\section{Preliminaries and fundamentals}

This section is devoted to presenting some preliminaries of the fractional calculus theory. In addition, some properties of Chebyshev polynomials of the second kind and their shifted ones are also presented.

\subsection{Some properties of Chebyshev polynomials of the second kind}

Chebyshev polynomials of the second kind $U_{j}(t), t \in[-1,1]$, Luke [Luke (1969)] have the following trigonometric definition:

$U_{j}(t)=\frac{\sin (j+1) \theta}{\sin \theta}, \quad t=\cos \theta$.

These polynomials may be generated with the aid of the recurrence relation:

$U_{j}(t)=2 t U_{j-1}(t)-U_{j-2}(t), \quad j \geq 2, \quad U_{0}(t)=1, U_{1}(t)=2 t$.

$U_{j}(t), j \geq 0$ are orthogonal on $[-1,1]$, in the sense that

$\int_{-1}^{1} \sqrt{1-t^{2}} U_{i}(t) U_{j}(t) d t= \begin{cases}\frac{\pi}{2}, & i=j, \\ 0, & i \neq j .\end{cases}$

Several properties and relations concerned with $U_{j}(x)$ can be found in Mason et al. [Mason and Handscomb (2003).

Let $U_{j}^{*}(t)$ be the shifted Chebyshev polynomials of the second kind defined in $[0,1]$ by $U_{j}^{*}(t)=U_{j}(2 t-1)$. They may be generated with the aid of the recurrence relation:

$U_{j}^{*}(t)=2(2 t-1) U_{j-1}^{*}(t)-U_{j-2}^{*}(t), \quad j \geq 2, \quad U_{0}^{*}(t)=1, U_{1}^{*}(t)=2(2 t-1)$.

Of the useful formulas of $U_{j}^{*}(t)$ are its analytic formula and its inversion which can be deduced as special cases of the analytic and inversion formulas of the shifted Jacobi polynomials Rainville [Rainville (1960)]. The analytic formula is given explicitly by:

$U_{j}^{*}(x)=\sum_{r=0}^{j} \frac{(-1)^{j+r} 2^{2 r}(j+r+1) !}{(j-r) !(2 r+1) !} x^{r}, \quad j \geq 0$, 
and its inversion formula is given explicitly by:

$x^{r}=\frac{4 \Gamma\left(\frac{3}{2}+r\right)}{\sqrt{\pi}} \sum_{p=0}^{r} \frac{r !(p+1) !}{(r-p) ! p !(r+p+2) !} U_{p}^{*}(x), \quad r \geq 0$.

The orthogonality relation of $U_{j}^{*}(t), j \geq 0$ is given by

$$
\int_{0}^{1} \sqrt{t-t^{2}} U_{i}^{*}(t) U_{j}^{*}(t) d t= \begin{cases}\frac{\pi}{8}, & i=j \\ 0, & i \neq j .\end{cases}
$$

\subsection{Some definitions and properties of fractional calculus}

In the following, we state some elementary definitions and relations of the fractional calculus [Sabatier, Agrawal and Machado (2007); Oldham and Spanier (1974)].

Definition 1. The Riemann-Liouville fractional integral operator ${ }_{0} I_{t}^{\nu}$ of order $\nu$ on the usual Lebesgue space $L_{1}[0,1]$ is defined as: for all $t \in(0,1)$

$$
\left({ }_{0} I_{t}^{\nu} f\right)(t)= \begin{cases}\frac{1}{\Gamma(\nu)} \int_{0}^{t}(t-\tau)^{\nu-1} f(\tau) d \tau, & \nu>0, \\ f(t), & \nu=0 .\end{cases}
$$

Definition 2. The left side Riemann-Liouville fractional derivative of order $\nu>0$ is defined by

$\left(D_{*}^{\nu} f\right)(t)=\left(\frac{d}{d t}\right)^{n}\left({ }_{0} I_{t}^{n-\nu} f\right)(t), n-1 \leq \nu<n, \quad n \in \mathbb{N}$.

Definition 3. The fractional differential operator in Caputo sense is defined as

$$
\left(D^{\nu} f\right)(t)=\frac{1}{\Gamma(n-\nu)} \int_{0}^{t}(t-\tau)^{n-\nu-1} f^{(n)}(\tau) d \tau, \nu>0, t>0,
$$

where $n-1 \leq \nu<n, n \in \mathbb{N}$.

The following properties are fulfilled by the operator $D^{\nu}$ for $n-1 \leq \nu<n$,

$$
\begin{aligned}
\left(D^{\nu} I^{\nu} f\right)(t) & =f(t), \\
\left(I^{\nu} D^{\nu} f\right)(t) & =f(t)-\sum_{k=0}^{n-1} \frac{f^{(k)}\left(0^{+}\right)}{k !}(t-a)^{k}, t>0, \\
D^{\nu} t^{k} & =\frac{\Gamma(k+1)}{\Gamma(k+1-\nu)} t^{k-\nu}, \quad k \in \mathbb{N}, k \geq\lceil\nu\rceil,
\end{aligned}
$$

where $\lceil\nu\rceil$ is the smallest integer greater than or equal to $\nu$. 


\section{Fractional derivatives of shifted Chebyshev polynomials of the second kind in terms of their original polynomials}

In this section, we aim to establish a new formula which expresses the fractional derivatives of shifted Chebyshev polynomials of the second kind in terms of their original polynomials. We show that the derived formula generalizes the well-known formula of the integer derivatives of the shifted Chebyshev polynomials of the second kind.

Recall the definition of the generalized hypergeometric function

${ }_{p} F_{q}\left(\begin{array}{c}f_{1}, f_{2} \ldots, f_{p} \\ g_{1}, g_{2} \ldots, g_{q}\end{array} \mid x\right)=\sum_{\ell=0}^{\infty} \frac{\left(f_{1}\right)_{\ell}\left(f_{2}\right)_{\ell} \ldots\left(f_{p}\right)_{\ell}}{\left(g_{1}\right)_{\ell}\left(g_{2}\right)_{\ell} \ldots\left(g_{q}\right)_{\ell}} \frac{x^{\ell}}{\ell !}$

where $f_{1}, f_{2}, \ldots, f_{p}, g_{1}, g_{2}, \ldots, g_{q}$, are complex or real parameters, with $g_{i} \neq 0$, for all $1 \leq i \leq q$.

The regularized hypergeometric function ${ }_{p} \tilde{F}_{q}$ is defined as

${ }_{p} \tilde{F}_{q}\left(\begin{array}{c}f_{1}, f_{2} \ldots, f_{p} \\ g_{1}, g_{2} \ldots, g_{q}\end{array} \mid x\right)=\frac{1}{\Gamma\left(g_{1}\right) \Gamma\left(g_{2}\right) \cdots \Gamma\left(g_{q}\right)} p F_{q}\left(\begin{array}{c}f_{1}, f_{2} \ldots, f_{p} \\ g_{1}, g_{2} \ldots, g_{q}\end{array} \mid x\right)$.

The following lemma which gives a transformation between two certain types of hypergeometric functions is useful in the derivation of our desired fractional derivatives formula.

Lemma 1. For all positive integers $j, p, n$, and $j \geq p+n$, the following transformation formula holds

$$
\begin{aligned}
{ }_{4} \tilde{F}_{3}\left(\begin{array}{c}
1,-j+n, 2+j+n, \frac{3}{2} \\
\frac{3}{2}+n, 1-p, 3+p
\end{array} \mid 1\right)= & \frac{\left(\frac{3}{2}\right)_{p}(-j+n)_{p}(2+j+n)_{p}}{(2 p+2) ! \Gamma\left(\frac{3}{2}+p+n\right)} \times \\
& { }_{3} F_{2}\left(\begin{array}{c}
-j+p+n, \frac{3}{2}+p, 2+j+p+n \\
3+2 p, \frac{3}{2}+p+n
\end{array}\right.
\end{aligned}
$$

Proof. The terminating hypergeometric series in the left hand side of (10) is given by

$$
{ }_{4} \tilde{F}_{3}\left(\begin{array}{c}
1,-j+n, 2+j+n, \frac{3}{2} \\
\frac{3}{2}+n, 1-p, 3+p
\end{array} \mid 1\right)=\sum_{\ell=p}^{j-n} \frac{\left(\frac{3}{2}\right)_{\ell}(-j+n)_{\ell}(2+j+n)_{\ell}}{(\ell-p) !(p+\ell+2) ! \Gamma\left(\frac{3}{2}+\ell+n\right)}
$$


Now, performing some computations on the right hand side of (11) enables one to write

$$
\begin{aligned}
& { }_{4} \tilde{F}_{3}\left(\begin{array}{c}
1,-j+n, 2+j+n, \frac{3}{2} \\
\frac{3}{2}+n, 1-p, 3+p
\end{array} \mid 1\right)=\sum_{\ell=0}^{j-n-p} \frac{\left(\frac{3}{2}\right)_{p+\ell}(-j+n)_{p+\ell}(2+j+n)_{p+\ell}}{\ell !(2 p+\ell+2) ! \Gamma\left(\frac{3}{2}+p+n+\ell\right)} \\
& =\frac{\left(\frac{3}{2}\right)_{p}(-j+n)_{p}(2+j+n)_{p}}{(2 p+2) ! \Gamma\left(\frac{3}{2}+p+n\right)} \times \\
& \sum_{\ell=0}^{j-n-p} \frac{\left(\frac{3}{2}+p\right)_{\ell}(-j+p+n)_{\ell}(2+j+p+n)_{\ell}}{\ell !(3+2 p)_{\ell}\left(\frac{3}{2}+p+n\right)_{\ell}} \\
& =\frac{\left(\frac{3}{2}\right)_{p}(-j+n)_{p}(2+j+n)_{p}}{(2 p+2) ! \Gamma\left(\frac{3}{2}+p+n\right)} \times \\
& { }_{3} F_{2}\left(\begin{array}{c|c}
-j+p+n, \frac{3}{2}+p, 2+j+p+n & 1 \\
3+2 p, \frac{3}{2}+p+n & 1
\end{array}\right) \text {. }
\end{aligned}
$$

The proof of Lemma 1 is now complete.

Theorem 1. The fractional derivatives of the shifted Chebyshev polynomials of the second kind can be approximated in terms of their original polynomials by the following formula:

$$
\begin{aligned}
D^{\theta} U_{j}^{*}(t) \simeq & \frac{2(-1)^{j+n}(j+n+1) ! \Gamma\left(\frac{3}{2}+n-\theta\right)}{(j-n) !} \times \\
& \sum_{p=0}^{N_{1}}(p+1){ }_{4} \tilde{F}_{3}\left(\begin{array}{c}
1,-j+n, 2+j+n, \frac{3}{2}+n-\theta \\
\frac{3}{2}+n, 1+n-p-\theta, 3+n+p-\theta
\end{array} \mid 1\right) U_{p}^{*}(t),
\end{aligned}
$$

where $N_{1}$ is a sufficiently large positive number, and $n=\lceil\theta\rceil$.

Proof. Based on the power form representation of $U_{j}^{*}(t)$ in (3) together with relation (9) yield

$$
D^{\theta} U_{j}^{*}(t)=\frac{1}{2} \sqrt{\pi} \sum_{\ell=\lceil\theta\rceil}^{j} \frac{(-1)^{j+\ell}(j+\ell+1) !}{(j-\ell) ! \Gamma\left(\frac{3}{2}+\ell\right) \Gamma(1+\ell-\theta)} x^{\ell-\theta} .
$$

Now, based on the inversion formula of $U_{j}^{*}(t)$ in (4), and for sufficiently large positive number $N_{1}$, we can approximate $x^{\ell-\theta}$ in the form

$$
x^{\ell-\theta} \simeq \frac{4 \Gamma\left(\ell-\theta+\frac{3}{2}\right)}{\sqrt{\pi}} \sum_{p=0}^{N_{1}} \frac{(1+p) !(\ell-\theta) !}{p !(\ell-p-\theta) !(2+\ell+p-\theta) !} U_{p}^{*}(t) .
$$


Inserting (14) into (13) leads to the following approximation for $D^{\theta} U_{j}^{*}(t)$

$$
\begin{aligned}
D^{\theta} U_{j}^{*}(t) & \simeq \frac{1}{2} \sqrt{\pi} \sum_{\ell=\lceil\theta\rceil}^{j} \frac{(-1)^{j+\ell}(j+\ell+1) ! 2^{1-2 \ell+2 \theta}}{(j-\ell) ! \Gamma\left(\frac{3}{2}+\ell\right) \Gamma(1+\ell-\theta)} \\
& \times \sum_{p=0}^{N_{1}} \frac{(1+p) \Gamma(2+2 \ell-2 \theta)}{\Gamma(1-p+\ell-\theta) \Gamma(3+p+\ell-\theta)} U_{p}^{*}(t) .
\end{aligned}
$$

After expanding the right hand side of relation (15) and rearranging the terms, we get

$$
\begin{aligned}
& D^{\theta} U_{j}^{*}(t) \simeq 2 \sum_{s=0}^{N_{1}}(s+1) \\
& \times \sum_{p=0}^{j-n} \frac{(-1)^{j+p+n}(j+p+n+1) ! \Gamma\left(\frac{3}{2}+p+n-\theta\right)}{(j-p-n) ! \Gamma\left(\frac{3}{2}+p+n\right) \Gamma(1+p+n-s-\theta) \Gamma(3+p+n+s-\theta)} U_{s}^{*}(t) .
\end{aligned}
$$

The last relation can be written in terms of hypergeometric form as

$$
\begin{aligned}
D^{\theta} U_{j}^{*}(t) \simeq & \frac{2(-1)^{j+n}(j+n+1) ! \Gamma\left(\frac{3}{2}+n-\theta\right)}{(j-n) !} \\
& \times \sum_{p=0}^{N_{1}}(p+1){ }_{4} \tilde{F}_{3}\left(\begin{array}{c}
1,-j+n, 2+j+n, \frac{3}{2}+n-\theta \\
\frac{3}{2}+n, 1+n-p-\theta, 3+n+p-\theta
\end{array}\right.
\end{aligned}
$$

The proof of Theorem 1 is now complete.

Corollary 1. Let $n$ be any positive integer. The $n t h$-derivative of the shifted Chebyshev polynomials of the second kind has the following representation in terms of their original polynomials

$$
D^{n} U_{j}^{*}(t)=2^{2 n} \sum_{\substack{p=0 \\(j+p+n) \text { even }}}^{j-n} \frac{(1+p)(n)_{\frac{1}{2}(j-p-n)}}{\left(\frac{1}{2}(j-p-n)\right) !\left(\frac{1}{2}(2+j+p+n)\right)_{1-n}} U_{p}^{*}(t) .
$$

Proof. If we set $\theta=n$ in relation (12), then it turns into

$$
\begin{aligned}
D^{n} U_{j}^{*}(t)= & \frac{(-1)^{j+n} \sqrt{\pi}(j+n+1) !}{(j-n) !} \sum_{p=0}^{j-n}(1+p) \times \\
& { }_{4} \tilde{F}_{3}\left(\begin{array}{c|c}
1,-j+n, 2+j+n, \frac{3}{2} & 1 \\
\frac{3}{2}+n, 1-p, 3+p & 1
\end{array}\right) U_{p}^{*}(x) .
\end{aligned}
$$

The transformation stated in Lemma 1 enables one to express the $n t h$-derivative in the 
following expression

$$
\begin{aligned}
D^{n} U_{j}^{*}(t)= & \frac{(-1)^{j+n} \sqrt{\pi}(1+j+n) !}{(j-n) !} \sum_{p=0}^{j-n} \frac{(1+p) !\left(\frac{3}{2}\right)_{p}(-j+n)_{p}(2+j+n)_{p}}{p !(2+2 p) ! \Gamma\left(\frac{3}{2}+n+p\right)} \times \\
& { }_{3} F_{2}\left(\begin{array}{c}
-j+p+n, \frac{3}{2}+p, 2+j+p+n \\
3+2 p, \frac{3}{2}+p+n
\end{array} \mid 1\right) U_{p}^{*}(t) .
\end{aligned}
$$

The ${ }_{3} F_{2}(1)$ in relation (19) can be reduced with the aid of Watson's identity to give

$$
\begin{aligned}
& { }_{3} F_{2}\left(\begin{array}{c}
\left.-j+p+n, \frac{3}{2}+p, 2+j+p+n \mid 1\right)= \\
3+2 p, \frac{3}{2}+p+n
\end{array} \mid \begin{array}{cl}
\Gamma\left(\frac{1}{2}(1+j-p-n)\right)(n)_{\frac{1}{2}(j-p-n)} & (j+p+n) \text { even, } \\
\frac{\sqrt{\pi}(2+p)_{\frac{1}{2}(j-p-n)}\left(\frac{3}{2}+p+n\right)_{\frac{1}{2}(j-p-n)}}{2,} & (j+p+n) \text { odd },
\end{array}\right.
\end{aligned}
$$

and therefore, we get

$$
D^{n} U_{j}^{*}(t)=2^{2 n} \sum_{\substack{p=0 \\(j+p+n) \text { even }}}^{j-n} \frac{(1+p)(n)_{\frac{1}{2}(j-p-n)}}{\left(\frac{1}{2}(j-p-n)\right) !\left(\frac{1}{2}(2+j+p+n)\right)_{1-n}} U_{p}^{*}(t) .
$$

Remark 1. Relation (17) can be written in the following alternative form

$$
D^{n} U_{j}^{*}(t)=\sum_{p=0}^{j-n} B_{p, j, n} U_{p}^{*}(t)
$$

where

$B_{p, j, n}=\frac{2^{2 n}(1+p)(n)_{\frac{1}{2}(j-p-n)} \xi_{p, j, n}}{\left(\frac{1}{2}(j-p-n)\right) !\left(\frac{1}{2}(2+j+p+n)\right)_{1-n}}$,

$\xi_{p, j, n}$ is given by

$\xi_{p, j, n}= \begin{cases}1, & (p+j+n) \text { even }, \\ 0, & \text { otherwise. }\end{cases}$

\section{Spectral solution of Riccati equation}

In this section, we are confined with finding a semi-analytic solution for the fractional-order Riccati differential equation with polynomial coefficients. An explicit second kind Chebyshev tau method (ES2CTM) is developed. Before proceeding in our algorithm, the following two lemmas are needed in the sequel. 
Lemma 2. For all nonnegative integers $k$ and $r$, the following linearization formula holds for shifted Chebyshev polynomials of the second kind [Askey (1975)].

$U_{k}^{*}(t) U_{r}^{*}(t)=\sum_{j=0}^{\min (k, r)} U_{k+r-2 j}^{*}(t)$.

Lemma 3. Let $i$ and $m$ be any two nonnegative integers. The moments formula for the shifted Chebyshev polynomials of the second kind is given explicitly by

$t^{i} U_{m}^{*}(t)=\frac{1}{2^{2 i}} \sum_{\ell=i-m}^{i+m}\left(\begin{array}{c}2 i \\ m+i-\ell\end{array}\right) U_{\ell}^{*}(t)$.

Proof. Lemma 3 can be proved based on making use of the recurrence relation (2) via induction after some lengthy straightforward steps.

Now, consider the following fractional Riccati differential equation with polynomial coefficients which generalizes the model considered in Raja et al. [Raja, Manzar and Samar (2015); Yüzbaşı (2013); Ezz-Eldien, Machado, Wang et al. (2019); Keshavarz, Ordokhani and Razzaghi (2014); Abd-Elhameed and Youssri (2014a)]:

$D^{\theta} y(t)+\left(\sum_{i=0}^{P} p_{i} t^{i}\right) y(t)+\left(\sum_{i=0}^{Q} q_{i} t^{i}\right) y^{2}(t)=S(t), \quad t \in(0,1)$,

subject to the initial condition

$y(0)=y_{0}$,

where $0<\theta \leq 1$ and $S(t)$ is a continuous known source function. We assume an approximate solution of the form

$y(t) \approx y_{N}(t)=\sum_{k=0}^{N} a_{k} U_{k}^{*}(t)$.

The residual of Eq. (25) is given by

$$
\begin{aligned}
R(t)= & \sum_{k=0}^{N} a_{k} D^{\theta} U_{k}^{*}(t)+\sum_{k=0}^{N} \sum_{i=0}^{P} p_{i} a_{k} t^{i} U_{k}^{*}(t)+\sum_{k, r=0}^{N} \sum_{i=0}^{Q} a_{k} a_{r} q_{i} t^{i} U_{k}^{*}(t) U_{r}^{*}(t) \\
& -\sum_{i=0}^{N} s_{i} U_{i}^{*}(t),
\end{aligned}
$$

where $s_{i}$ are the Fourier second-kind Chebyshev coefficients of $S(t)$. Now Theorem 1 enables one to write the expression

$D^{\theta} U_{k}^{*}(t)=\sum_{i=0}^{N_{1}} \gamma_{i, k, \theta} U_{i}^{*}(t)$ 
where $\gamma_{i, k, \theta}$ is given by

$$
\begin{aligned}
\gamma_{i, k, \theta} & =\frac{2(-1)^{k+n}(i+1)(1+k+n) ! \Gamma\left(\frac{3}{2}+n-\theta\right)}{(k-n) !} \\
& \times \tilde{F}_{3}\left(\begin{array}{c}
1,-k+n, 2+k+n, \frac{3}{2}+n-\theta \\
\frac{3}{2}+n, 1+n-i-\theta, 3+n+i-\theta
\end{array}\right.
\end{aligned}
$$

In virtue of (28) and the linearization formula (23), the residual in (27) can be transformed into

$$
\begin{aligned}
R(t)= & \sum_{k=0}^{N} \sum_{i=0}^{N_{1}} \gamma_{i, k, \theta} a_{k} U_{i}^{*}(t)+\sum_{k=0}^{N} \sum_{i=0}^{P} p_{i} a_{k} t^{i} U_{k}^{*}(t) \\
& +\sum_{k, r=0}^{N} \sum_{i=0}^{Q} \sum_{j=0}^{\min (k, r)} a_{k} a_{r} q_{i} t^{i} U_{k+r-2 j}^{*}(t)-\sum_{i=0}^{N} s_{i} U_{i}^{*}(t) .
\end{aligned}
$$

With the aid of Lemma 3, the moments formula of $U_{m}^{*}(t)$ can be written in the form

$t^{i} U_{m}^{*}(t)=\sum_{\ell=i-m}^{i+m} \mu_{i, m, \ell} U_{\ell}^{*}(t)$,

and

$$
\mu_{i, m, \ell}=\frac{1}{2^{2 i}}\left(\begin{array}{c}
2 i \\
m+i-\ell
\end{array}\right) \text {. }
$$

Now, the residual in Eq. (30) takes the following form

$$
\begin{aligned}
R(t) & =\sum_{k=0}^{N} \sum_{i=0}^{N_{1}} a_{k} \gamma_{i, k, \theta} U_{i}^{*}(t)+\sum_{k=0}^{N} \sum_{i=0}^{P} \sum_{\ell=i-k}^{i+k} a_{k} p_{i} \mu_{i, k, \ell} U_{\ell}^{*}(t) \\
& +\sum_{k, r=0}^{N} \sum_{i=0}^{Q} \sum_{j=0}^{\min (k, r)} \sum_{\ell=i-k-r+2 j}^{i+k+r-2 j} a_{k} a_{r} q_{i} \mu_{i, k+r-2 j, \ell} U_{\ell}^{*}(t)-\sum_{i=0}^{N} s_{i} U_{i}^{*}(t) .
\end{aligned}
$$

The application of the typical tau method yields

$$
\int_{0}^{1} \sqrt{t-t^{2}} R(t) U_{m}^{*}(t) d t=0, \quad m=0, \ldots N-1 .
$$

Thanks to the orthogonality relation (5), relation (31) can be turned into

$$
\begin{aligned}
& \sum_{k=0}^{N} \sum_{i=0}^{N_{1}} a_{k} \gamma_{i, k, \theta} \delta_{i, m}+\sum_{k=0}^{N} \sum_{i=0}^{P} \sum_{\ell=i-k}^{i+k} a_{k} p_{i} \mu_{i, k, \ell} \delta_{\ell, m} \\
& +\sum_{k, r=0}^{N} \sum_{i=0}^{Q} \sum_{j=0}^{\min (k, r)} \sum_{\ell=i-k-r+2 j}^{i+k+r-2 j} a_{k} a_{r} q_{i} \mu_{i, k+r-2 j, \ell} \delta_{\ell, m}=\sum_{i=0}^{N} s_{i} \delta_{i, m},
\end{aligned}
$$


and $\delta_{i, j}$ is the Kronecker delta function.

In addition, the initial condition (26), yields

$\sum_{k=0}^{N}(-1)^{k}(k+1) a_{k}=y_{0}$.

Finally Eqs. (32)-(33) generate an algebraic system in the unknown expansion coefficients $a_{k}$ of quadratic nonlinearity, we solve this system with Newton's iterative method "FindRoot" package in Mathematica 12, with the initial guess $a_{k}=10^{-k}$, hence the approximate semi-analytic solution can be obtained.

\section{Investigation of the convergence and error analysis}

Note 1. By writing $a_{k} \lesssim b_{k}$, we mean that there exists a positive generic constant $C$ such that $a_{k} \leq C b_{k}$

Lemma 4. [Mason and Handscomb (2003)] For all $k \geq 0$, we have the following estimate $\left|U_{k}^{*}(t)\right| \leq k+1$.

Lemma 5. For all $k \geq 0$, we have the following estimate $\left|D^{\theta} U_{k}^{*}(t)\right| \lesssim k^{\theta+1}$.

\section{Proof.}

$D^{\theta} U_{j}^{*}(t)=\sum_{r=0}^{j} \frac{(-1)^{j+r} 2^{2 r}(j+r+1) ! r !}{(j-r) !(2 r+1) ! \Gamma(r+1-\theta)} t^{r-\theta}$,

using the Legendre duplication formula and noting that $0<t<1$, we get the desired result.

Theorem 2. If $y(t) \in C^{n}[0,1]$ for some $n>3$, then we have the following estimates:

$\left|a_{k}\right| \lesssim \frac{1}{k^{n}}, \quad$ for all $\quad k>n$,

$\left|y(t)-y_{N}(t)\right| \lesssim \frac{1}{N^{n-2}} \quad$ for all $\quad k>n$.

Proof. Following similar procedures to those followed in Abd-Elhameed et al. [Abd-Elhameed and Youssri (2014b)], we get

$a_{i}=\frac{8}{\pi} \int_{0}^{1} \sqrt{t-t^{2}} y(t) U_{i}^{*}(t) d t$

If we substitute by $2 t-1=\cos \gamma$ in (37), then we get

$$
a_{i}=\frac{2}{\pi} \int_{0}^{\pi} y\left(\frac{1+\cos \gamma}{2}\right) \sin \gamma \sin (i+1) \gamma d \gamma
$$


which gives after integration by parts $n$ times

$a_{i}=\frac{1}{8 \pi} \int_{0}^{\pi} y^{(n)}\left(\frac{1+\cos \gamma}{2}\right) \Omega_{i}(\gamma) d \gamma$,

where $\Omega_{i}(\gamma)$ is a trigonometric polynomial in $\cos \gamma, \sin \gamma$. Now noting that $|\cos \gamma| \leq$ $1,|\sin \gamma| \leq 1$ and after some manipulations we get the desired result. For the second part of the theorem, just noting the well known inequality: $\sum_{k=N+1}^{\infty} f(k) \leqslant \int_{N}^{\infty} f(x) d x$, where $f(k)=a_{k}$, we directly get the result.

In the next theorem, we find an upper estimate for the following global error

$\mathcal{R}_{N}(t)=D^{\theta} y_{N}(t)+\left(\sum_{i=0}^{P} p_{i} t^{i}\right) y_{N}(t)+\left(\sum_{i=0}^{Q} q_{i} t^{i}\right) y_{N}^{2}(t)-S(t)$.

Theorem 3. If $y(t)$ satisfies the hypothesis of Theorem 2 and $|y(t)| \leq M$, for some positive constant $M$, let $\tilde{P}=\sum_{i=0}^{P}\left|p_{i}\right|, \tilde{Q}=\sum_{i=0}^{Q}\left|q_{i}\right|$ are positive numbers, then we have the following global error estimates

$\left|\mathcal{R}_{N}(t)\right| \lesssim \frac{1}{N^{n-\theta-1}}+\frac{\tilde{P}+2 M \tilde{Q}}{N^{n-2}}$.

Proof. The approximate solution $y_{N}(t)$ satisfies

$D^{\theta} y_{N}(t)+\left(\sum_{i=0}^{P} p_{i} t^{i}\right) y_{N}(t)+\left(\sum_{i=0}^{Q} q_{i} t^{i}\right) y_{N}^{2}(t) \approx S(t)$.

From Eq. (25), we have

$\mathcal{R}_{N}(t)=D^{\theta}\left(y(t)-y_{N}(t)\right)+\left(\sum_{i=0}^{P} p_{i} t^{i}\right)\left(y(t)-y_{N}(t)\right)+\left(\sum_{i=0}^{Q} q_{i} t^{i}\right)\left(y^{2}(t)-y_{N}^{2}(t)\right)$.

Now

$$
\begin{aligned}
\left|\mathcal{R}_{N}(t)\right| & \leq\left|D^{\theta}\left(y(t)-y_{N}(t)\right)\right|+\tilde{P}\left|y(t)-y_{N}(t)\right|+\tilde{Q}\left|y^{2}(t)-y_{N}^{2}(t)\right| \\
& \leq\left|\sum_{k=N+1}^{\infty}\right| a_{k} D^{\theta} U_{k}^{*}(t) \mid+\tilde{P} / N^{n-2}+2 M \tilde{Q} / N^{n-2} .
\end{aligned}
$$

With the aid of the two Lemmas 4, 5, and by the properties of the Riemann-zeta function, we have

$\left|\mathcal{R}_{N}(t)\right| \lesssim 1 / N^{n-\theta-1}+\tilde{P} / N^{n-2}+2 M \tilde{Q} / N^{n-2}$,

which completes the proof of the theorem. 


\section{Numerical experiments}

In this section, we illustrate the applicability, efficiency and accuracy of the presented algorithm by four numerical examples, we compare our results with those obtained using some other techniques.

Example 1. Consider the following nonlinear fractional Ricatti equation [Kashkari and Syam (2016); Bota and Căruntu (2017); Ezz-Eldien, Machado, Wang et al. (2019)]

$D^{\theta} y(t)+y(t)+y^{2}(t)=t^{\frac{3}{2}}\left(\frac{8}{3 \Gamma(0.5)}+t^{\frac{1}{2}}+t^{\frac{5}{2}}\right), \quad t \in[0,1]$

$y(0)=0$,

If $\theta=\frac{1}{2}$, the exact smooth solution of Eq. (38) is given by $y(t)=t^{2}$. We handle this problem in two cases:

- Case 1: If $\theta=\frac{1}{2}$, we have an exact analytic solution.

- Case 2: If $\theta \in(0,1), \theta \neq \frac{1}{2}$. there is no exact solution.

For case 1, we apply our algorithm with $N=4$, and solve the algebraic system in (32)-(33) to get

$a_{0}=0.3125, a_{1}=0.25, a_{2}=0.0625, a_{3}=a_{4}=0$,

and consequently, the approximate solution $y_{4}(x)$ can be explicitly calculated

$$
\begin{aligned}
y_{4}(t)= & 0.3125+0.25(-2+4 t)+0.0625\left(3-16 t+16 t^{2}\right) \\
& +0\left(-4+40 t-96 t^{2}+64 t^{3}\right)+0\left(5-80 t+336 t^{2}-512 t^{3}+256 t^{4}\right)=t^{2},
\end{aligned}
$$

which is the exact solution.

For case 2, in Tab. 1, due to the nonavailability of the exact solution for $\theta<1$, we list the maximum residual error

$\epsilon_{N}=\max _{0 \leq t \leq 1}\left|D^{\theta} y_{N}(t)+y_{N}(t)+y_{N}^{2}(t)-t^{\frac{3}{2}}\left(\frac{8}{3 \Gamma(0.5)}+t^{\frac{1}{2}}+t^{\frac{5}{2}}\right)\right|$,

for the case $N=4$ and for different values of $\theta$.

Table 1: The maximum residual errors for Example 1

\begin{tabular}{cccccc}
\hline$\theta$ & 0.1 & 0.3 & 0.5 & 0.7 & 0.9 \\
\hline$\epsilon_{N}$ & $2.01 E^{-5}$ & $3.58 E^{-6}$ & 0.00 & $2.39 E^{-7}$ & $1.17 E^{-7}$ \\
\hline
\end{tabular}

Example 2. Consider the following nonlinear fractional Ricatti equation with variable coefficients

$D^{\theta} y(t)+t(1+y(t))^{2}=t(t+2)^{2}+\frac{2 \sqrt{t}}{\sqrt{\pi}}, \quad t \in[0,1]$,

$y(0)=1$,

if $\theta=\frac{1}{2}$, the exact smooth solution of Eq. (39) is $y(t)=1+t$. We handle this problem in two cases: 
- Case 1: If $\theta=\frac{1}{2}$, we have an exact analytic solution.

- Case 2: If $\theta \in(0,1), \theta \neq \frac{1}{2}$. there is no exact solution.

For case 1, we apply our algorithm with $N=3$, then we obtain the following system of equations

$$
\left\{\begin{array}{l}
\frac{a_{0}-2 a_{1}+3 a_{2}-4 a_{3}=1}{1575 \sqrt{\pi}} \\
+\frac{\pi}{256}\left(16 a_{0}^{2}+16\left(a_{1}+2\right) a_{0}+16 a_{1}^{2}+16 a_{2}^{2}+16 a_{3}^{2}+16 a_{1}\left(a_{2}+1\right)+16 a_{2} a_{3}-95\right)=0 \\
\frac{16\left(396 a_{1}+1232 a_{2}-648 a_{3}-99\right)}{10395 \sqrt{\pi}}+\frac{\pi}{256}\left(8 a_{0}^{2}+16\left(2 a_{1}+a_{2}+1\right) a_{0}\right. \\
\left.+16 a_{1}^{2}+16 a_{2}^{2}+16 a_{3}^{2}+16 a_{2}+32 a_{2} a_{3}+16 a_{1}\left(2 a_{2}+a_{3}+2\right)-63\right)=0 \\
\frac{8\left(-572 a_{1}+5616 a_{2}+11176 a_{3}+143\right)}{45045 \sqrt{\pi}} \\
+\frac{\pi}{256}\left(16 a_{1}^{2}+16\left(a_{0}+2 a_{2}+2 a_{3}+1\right) a_{1}+16 a_{2}^{2}+16 a_{3}^{2}\right. \\
\left.+16 a_{0} a_{3}+16 a_{3}+32 a_{2}\left(a_{0}+a_{3}+1\right)-11\right)=0 .
\end{array}\right.
$$

The system in (40) can be solved to give

$a_{0}=1.5, a_{1}=0.25, a_{2}=a_{3}=0$,

and consequently

$y_{3}(t)=1.5+0.25(-2+4 t)+\left(3-16 t+16 t^{2}\right)+0\left(-4+40 t-96 t^{2}+64 t^{3}\right)=1+t$,

which is the exact solution.

For case 2, in Tab. 2, due to the nonavailability of the exact solution for $\theta<1$, we list the maximum residual error

$\epsilon_{N}=\max _{0 \leq t \leq 1}\left|D^{\theta} y_{N}(t)+t\left(1+y_{N}(t)\right)^{2}-t(t+2)^{2}-\frac{2 \sqrt{t}}{\sqrt{\pi}}\right|$,

for the case $N=3$ and for different values of $\theta$.

Table 2: The maximum residual errors for Example 2

\begin{tabular}{cccccc}
\hline$\theta$ & 0.1 & 0.3 & 0.5 & 0.7 & 0.9 \\
\hline$\epsilon_{N}$ & $3.24 E^{-4}$ & $5.27 E^{-5}$ & 0.00 & $7.25 E^{-6}$ & $2.16 E^{-6}$ \\
\hline
\end{tabular}

Example 3. Consider the following nonlinear fractional Ricatti equation [Yüzbaş1 (2013); Raja, Manzar and Samar (2015); Ezz-Eldien, Machado, Wang et al. (2019); Keshavarz, Ordokhani and Razzaghi (2014); Abd-Elhameed and Youssri (2014a)]

$D^{\theta} y(t)+y^{2}(t)=1, \quad t \in[0,1], 0<\theta \leq 1$,

$y(0)=0$, 


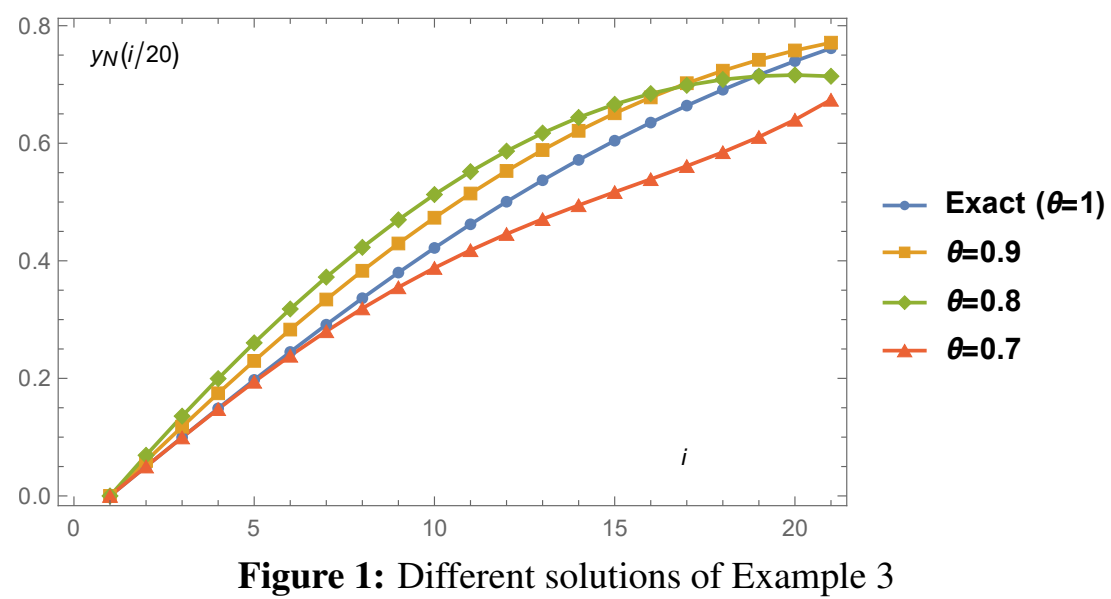

when $\theta=1$, the exact smooth solution of (41) is $y(t)=\tanh t$. Many authors handles Eq. (41). For example, Yuzbasi in [Yüzbaşı (2013)] applied the collocation method based on Bernstein polynomials, Raja et al. [Raja, Manzar and Samar (2015)] adopted the sequential quadratic programming method and Ezz-Eldien et al. [Ezz-Eldien, Machado, Wang et al. (2019)] used the first-kind Chebyshev polynomials. We compare our method, namely, $E S 2 C T M$ with some previous mentioned methods for the case $\theta=1$ in Tab. 3. In Fig. 1 , we analyze the effect of $\theta$ on the behavior of the solution using the "ListPlot" command from Mathematica, we plot $y_{N}(i / 20), 0 \leq i \leq 20$ for different values of $\theta$.

Table 3: Comparison between different algorithms for Example 3 for $\theta=2$

\begin{tabular}{cccccc}
\hline$x$ & Exact & [Yüzbaş1 (2013)] & [Raja, Manzar and Samar (2015)] & [Ezz-Eldien, Machado, Wang et al. (2019)] & ES2CTM $N=12$ \\
\hline 0.0 & 0.00000000000 & 0.000000000000 & 0.0000000011 & 0.000000000000 & 0.000000000000 \\
0.2 & 0.19737532022 & 0.197375320493 & 0.1973918880 & 0.19737532017 & 0.19737532024 \\
0.4 & 0.37994896226 & 0.379948962506 & 0.3799632287 & 0.379948962207 & 0.37994896229 \\
0.6 & 0.53704956700 & 0.537049567214 & 0.5370622335 & 0.53704956701 & 0.53704956714 \\
0.8 & 0.66403677027 & 0.664036770562 & 0.6640456511 & 0.66403677030 & 0.66403677024 \\
1.0 & 0.76159415596 & 0.761594224400 & 0.7616019763 & 0.76159415595 & 0.76159415594 \\
\hline
\end{tabular}

Example 4. Consider the following nonlinear fractional Ricatti equation [Yüzbaş1 (2013); Li, Sun, Zheng et al. (2014); Raja, Manzar and Samar (2015); Ezz-Eldien, Machado, Wang et al. (2019); Keshavarz, Ordokhani and Razzaghi (2014); Odetunde and Taiwo (2014); Abd-Elhameed and Youssri (2014a)]

$$
\begin{aligned}
& D^{\theta} y(t)-y^{2}(t)=1, \quad t \in[0,1], 0<\theta \leq 1, \\
& y(0)=0,
\end{aligned}
$$

when $\theta=1$, the exact smooth solution of $(42)$ is $y(t)=\tan t$. Many authors handles Eq. (42), for example, Li et al. [Li, Sun, Zheng et al. (2014)] used the Haar wavelet operational matrix method, Odetunde and Taiwo in Odetunde et al. [Odetunde and Taiwo (2014)] applied a decomposition algorithm and Ezz-Eldien et al. [Ezz-Eldien, Machado, Wang et al. (2019)] used the first-kind Chebyshev polynomials. We compare our method (ES2CTM) with the previous mentioned methods for the case $\theta=1$ in Tab. 4. In Fig. 2, we 


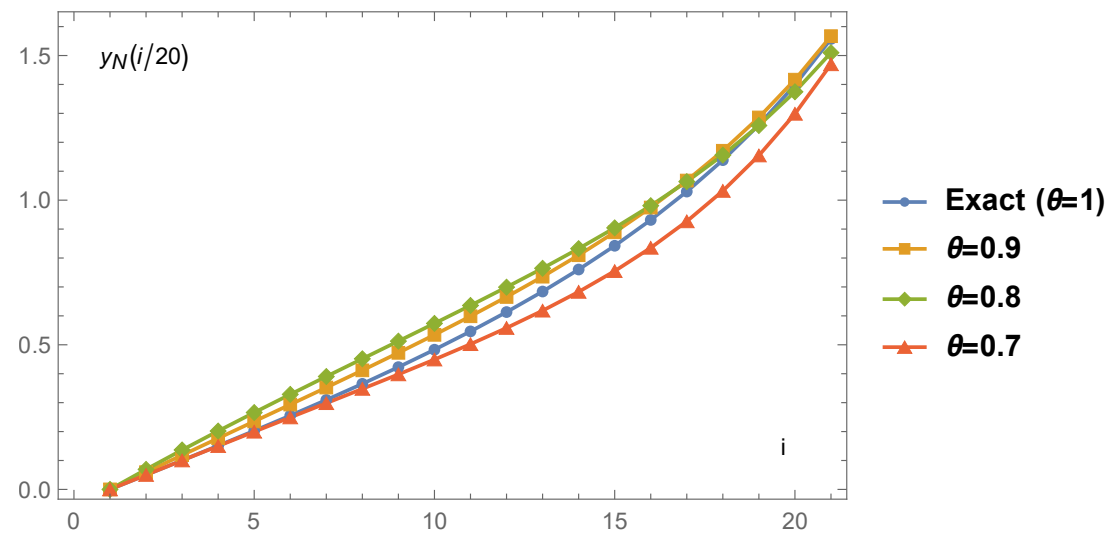

Figure 2: Different solutions of Example 4

analyze the effect of $\theta$ on the behavior of the solution using the "ListPlot" command from Mathematica, we plot $y_{N}(i / 20), 0 \leq i \leq 20$ for different values of $\theta$.

Table 4: Comparison between different algorithms for Example 4 for $\theta=1$

\begin{tabular}{cccccc}
\hline$x$ & Exact & [Li, Sun, Zheng et al. (2014)] & [Odetunde and Taiwo (2014)] & [Ezz-Eldien, Machado, Wang et al. (2019)] & ESKCM $N=12$ \\
\hline 0.0 & 0.00000000000 & & 0.0000000000 & 0.00000000000 & 0.00000000000 \\
0.1 & 0.10033467208 & 0.100342 & 0.1003346713 & 0.1003346714 & 0.10033467208 \\
0.2 & 0.20271003550 & 0.202726 & 0.2027099297 & 0.2027100349 & 0.20271003550 \\
0.3 & 0.30933624961 & 0.309372 & 0.3093343442 & 0.3093362509 & 0.30933624961 \\
0.4 & 0.42279321873 & 0.422832 & 0.4227777155 & 0.4227932186 & 0.42279321873 \\
0.5 & 0.54630248984 & 0.546363 & 0.5462212762 & 0.5463024891 & 0.54630248984 \\
0.6 & 0.68413680834 & 0.684251 & 0.6838056920 & 0.6841368110 & 0.68413680834 \\
0.7 & 0.84228838046 & 0.842411 & 0.8411449022 & 0.8422883779 & 0.84228838046 \\
0.8 & 1.02963855705 & 1.029849 & 1.0261001110 & 1.0296385599 & 1.02963855705 \\
0.9 & 1.26015821755 & 1.260573 & 1.2499664940 & 1.2601582184 & 1.26015821754 \\
1.0 & 1.55740772465 & 1.557938 & 1.5293009690 & 1.5574077258 & 1.55740772469 \\
\hline
\end{tabular}

Example 5. Consider the following nonlinear fractional Ricatti equation with variable coefficients

$D^{\theta} y(t)-t^{2} y(t)+t y^{2}(t)=\frac{2 \sqrt{t}}{\sqrt{\pi}}, \quad t \in[0,1], 0<\theta \leq 1$,

$y(0)=0$,

with the exact smooth solution $y(t)=t$. We apply our algorithm with $N=1$, to get the following system of algebraic equations:

$\frac{8\left(4 a_{1}-1\right)}{15 \sqrt{\pi}}+\frac{1}{128} \pi\left(8 a_{0}^{2}+\left(8 a_{1}-5\right) a_{0}+4 a_{1}\left(2 a_{1}-1\right)\right)=0$,

$a_{0}-2 a_{1}=0$,

which yields

$a_{0}=\frac{1}{2}, \quad a_{1}=\frac{1}{4}$,

and consequently

$y_{1}(t)=\frac{1}{4}(4 t-2)+\frac{1}{2}=t$, 
which is the exact solution.

Example 6. Consider the following nonlinear fractional Ricatti equation [Singh and Srivastava (2019)]

$$
\begin{aligned}
& D^{\theta} y(t)-1-2 y(t)+y^{2}(t)=0, \quad t \in[0,1], 0<\theta \leq 1, \\
& y(0)=0 .
\end{aligned}
$$

The exact smooth solution of (44) when $\theta=1$ is

$y(t)=1+\sqrt{2} \tanh \left(\sqrt{2} t+\frac{1}{2} \ln \left(\frac{\sqrt{2}-1}{\sqrt{2}+1}\right)\right)$.

We apply our algorithm with $N=15$, in Tab. 5, we compare between the best errors of Eq. (44) which resulted from our present method and the method proposed in singh et al. [Singh and Srivastava (2019)] for the case $\theta=1$, in Tab. 6, due to the nonavailability of the exact solution for $\theta<1$, we list the maximum residual error

$\epsilon_{N}=\max _{0 \leq t \leq 1}\left|D^{\theta} y_{N}(t)-1-2 y_{N}(t)+y_{N}^{2}(t)\right|$

for the case $N=15$ and for different values of $\theta$.

Table 5: Comparison between absolute errors for Example 6 for $\theta=1$

\begin{tabular}{ccc}
\hline$t$ & [Singh and Srivastava (2019)] & Present Method \\
\hline 0.1 & $4.57 E^{-9}$ & $2.22 E^{-15}$ \\
0.2 & $9.74 E^{-10}$ & $2.67 E^{-15}$ \\
0.3 & $3.71 E^{-9}$ & $1.01 E^{-14}$ \\
0.4 & $1.29 E^{-9}$ & $2.34 E^{-14}$ \\
0.5 & $1.93 E^{-9}$ & $3.27 E^{-14}$ \\
0.6 & $2.74 E^{-9}$ & $2.24 E^{-14}$ \\
0.7 & $4.32 E^{-9}$ & $4.68 E^{-14}$ \\
0.8 & $2.43 E^{-9}$ & $7.25 E^{-14}$ \\
0.9 & $3.59 E^{-10}$ & $3.67 E^{-14}$ \\
1.0 & $7.01 E^{-9}$ & $2.38 E^{-14}$ \\
\hline
\end{tabular}

Table 6: The maximum residual errors for Example 6

\begin{tabular}{cccccc}
\hline$\theta$ & 0.1 & 0.3 & 0.5 & 0.7 & 0.9 \\
\hline$\epsilon_{N}$ & $4.24 E^{-16}$ & $7.22 E^{-16}$ & $5.27 E^{-16}$ & $3.24 E^{-17}$ & $4.28 E^{-18}$ \\
\hline
\end{tabular}

\section{Concluding remarks}

Herein, a new formula for approximating the fractional-order derivative of the second-kind Chebyshev polynomials was derived and proved. This formula of course generalizes the well-known integer derivatives formula. The fractional derivatives formula was utilized to 
analyze explicit second-kind Chebyshev algorithm for solving the fractional-order Riccati differential equation. We do believe that the derived fractional formula in this paper is novel and it can be employed for solving some other types of fractional differential equations.

Acknowledgement: The authors would like to thank the referees for carefully reading the paper and also for their constructive and valuable comments which have improved the paper.

\section{References}

Abd-Elhameed, W.; Doha, E.; Youssri, Y.; Bassuony, M. (2016): New Tchebyshev-Galerkin operational matrix method for solving linear and nonlinear hyperbolic telegraph type equations. Numerical Methods for Partial Differential Equations, vol. 32, no. 6, pp. 1553-1571.

Abd-Elhameed, W.; Youssri, Y. (2014): New ultraspherical wavelets spectral solutions for fractional Riccati differential equations. Abstract and Applied Analysis, vol. 2014.

Abd-Elhameed, W.; Youssri, Y. (2014): Numerical solutions for Volterra-Fredholm-Hammerstein integral equations via second kind Chebyshev quadrature collocation algorithm. Advances in Mathematical Sciences and Applications, vol. 24, pp. 129-141.

Abd-Elhameed, W.; Youssri, Y. (2015): New spectral solutions of multi-term fractional order initial value problems with error analysis. Computer Modeling in Engineering \& Sciences, vol. 105, pp. 375-398.

Abd-Elhameed, W.; Youssri, Y. (2016a): A novel operational matrix of Caputo fractional derivatives of Fibonacci polynomials: spectral solutions of fractional differential equations. Entropy, vol. 18, no. 10, pp. 345.

Abd-Elhameed, W.; Youssri, Y. (2016b): Spectral solutions for fractional differential equations via a novel Lucas operational matrix of fractional derivatives. Romanian Journal of Physics, vol. 61, no. 5-6, pp. 795-813.

Abd-Elhameed, W.; Youssri, Y. (2018): Fifth-kind orthonormal Chebyshev polynomial solutions for fractional differential equations. Computational and Applied Mathematics, vol. 37, pp. 2897-2921.

Abd-Elhameed, W.; Youssri, Y. (2019): Sixth-kind Chebyshev spectral approach for solving fractional differential equations. International Journal of Nonlinear Sciences and Numerical Simulation, vol. 20, no. 2, pp. 191-203.

Ali, M.; Alquran, M.; Jaradat, I. (2019): Asymptotic-sequentially solution style for the generalized Caputo time-fractional Newell-Whitehead-Segel system. Advances in Difference Equations, vol. 2019, no. 1, pp. 70.

Alquran, M.; Jaradat, I. (2018): A novel scheme for solving Caputo time-fractional nonlinear equations: theory and application. Nonlinear Dynamics, vol. 91, no. 4, pp. 2389-2395. 
Anastassiou, G. A.; Argyros, I. K. (2016): Intelligent numerical methods: applications to fractional calculus. Springer.

Askey, R. (1975): Orthogonal polynomials and special functions. Odyssey Press, Dover, New Hampshire, USA.

Balaji, S. (2015): Legendre wavelet operational matrix method for solution of fractional order Riccati differential equation. Journal of the Egyptian Mathematical Society, vol. 23, no. 2, pp. 263-270.

Bota, C.; Căruntu, B. (2017): Analytical approximate solutions for quadratic Riccati differential equation of fractional order using the Polynomial Least Squares Method. Chaos, Solitons \& Fractals, vol. 102, pp. 339-345.

Daftardar-Gejji, V.; Jafari, H. (2007): Solving a multi-order fractional differential equation using Adomian decomposition. Applied Mathematics and Computation, vol. 189, no. 1, pp. 541-548.

Doha, E. (2002): On the coefficients of differentiated expansions and derivatives of Jacobi polynomials. Journal of Physics A: Mathematical and General, vol. 35, no. 15, pp. 3467.

Doha, E.; Abd-Elhameed, W.; Bassuony, M. (2015): On the coefficients of differentiated expansions and derivatives of Chebyshev polynomials of the third and fourth kinds. Acta Mathematica Scientia, vol. 35, no. 2, pp. 326-338.

Doha, E.; Abd-Elhameed, W.; Youssri, Y. (2013): Second kind Chebyshev operational matrix algorithm for solving differential equations of Lane-Emden type. New Astronomy, vol. 23, pp. 113-117.

Einicke, G.; White, L.; Bitmead, R. (2003): The use of fake algebraic Riccati equations for co-channel demodulation. IEEE Transactions on Signal Processing, vol. 51, no. 9, pp. 2288-2293.

Ezz-Eldien, S.; Machado, J.; Wang, Y.; Aldraiweesh, A. (2019): An algorithm for the approximate solution of the fractional Riccati differential equation. International Journal of Nonlinear Sciences and Numerical Simulation, vol. 20, no. 6, pp. 661-674.

Jena, R.; Chakraverty, S.; Baleanu, D. (2019): On the solution of an imprecisely defined nonlinear time-fractional dynamical model of marriage. Mathematics, vol. 7, no. 8, pp. 689.

Jena, R.; Chakraverty, S.; Jena, S. (2019): Dynamic response analysis of fractionally damped beams subjected to external loads using homotopy analysis method. Journal of Applied and Computational Mechanics, vol. 5, no. 2, pp. 355-366.

Kashkari, B.; Syam, M. (2016): Fractional-order Legendre operational matrix of fractional integration for solving the Riccati equation with fractional order. Applied Mathematics and Computation, vol. 290, pp. 281-291.

Keshavarz, E.; Ordokhani, Y.; Razzaghi, M. (2014): Bernoulli wavelet operational matrix of fractional order integration and its applications in solving the fractional order differential equations. Applied Mathematical Modelling, vol. 38, no. 24, pp. 6038-6051.

Khader, M. (2013): Numerical treatment for solving fractional Riccati differential equation. Journal of the Egyptian Mathematical Society, vol. 21, no. 1, pp. 32-37. 
Khan, N.; Ara, A.; Jamil, M. (2011): An efficient a pproach $\mathrm{f}$ or s olving the Riccati equation with fractional orders. Computers \& Mathematics with Applications, vol. 61, no. 9, pp. 2683-2689.

Khan, N.; Ara, A.; Khan, N. (2013): Fractional-order Riccati differential equation: analytical approximation and numerical results. Advances in Difference Equations, vol. 2013, no. 1, pp. 185.

Li, Y.; Sun, N.; Zheng, B.; Wang, Q.; Zhang, Y. (2014): Wavelet operational matrix method for solving the Riccati differential equation. Communications in Nonlinear Science and Numerical Simulation, vol. 19, no. 3, pp. 483-493.

Luke, Y. (1969): Special Functions and Their Approximations, volume 1. Academic press.

Masjed-Jamei, M. (2006): Some New Classes of Orthogonal Polynomials and Special Functions: A Symmetric Generalization of Sturm-Liouville Problems and its Consequences. $\mathrm{PhD}$ thesis, 2006.

Mason, J.; Handscomb, D. (2003): Chebyshev Polynomials. Chapman and Hall, New York, NY, CRC, Boca Raton.

Meerschaert, M.; Tadjeran, C. (2006): Finite difference approximations for two-sided space-fractional partial differential equations. Applied Numerical Mathematics, vol. 56, no. 1, pp. 80-90.

Odetunde, O.; Taiwo, O. (2014): A decomposition algorithm for the solution of fractional quadratic Riccati differential equations with Caputo derivatives. American Journal of Computational and Applied Mathematics, vol. 4, no. 3, pp. 83-91.

Odibat, Z.; Momani, S. (2008): Modified homotopy perturbation m ethod: application to quadratic Riccati differential equation of fractional order. Chaos Solitons Fractals, vol. 36, no. 1, pp. 167-174.

Oldham, K.; Spanier, J. (1974): The Fractional Calculus Theory and Applications of Differentiation and Integration to Arbitrary Order, volume 111. Elsevier.

Öztürk, Y.; Anapalı, A.; Gülsu, M.; Sezer, M. (2013): A collocation method for solving fractional Riccati differential equation. Journal of Applied Mathematics, vol. 2013.

Rainville, E. (1960): Special Functions. Macmillan Co., New York.

Raja, M.; Manzar, M.; Samar, R. (2015): An efficient c omputational intelligence approach for solving fractional order Riccati equations using ANN and SQP. Applied Mathematical Modelling, vol. 39, no. 10-11, pp. 3075-3093.

Rudolf, H. (2000): Applications of Fractional Calculus in Physics. World Scientific.

Sabatier, J.; Agrawal, O.; Machado, J. (2007): Advances in Fractional Calculus, volume 4. Springer.

Salehi, Y.; Darvishi, M. (2016): An investigation of fractional Riccati differential equation. Optik, vol. 127, no. 23, pp. 11505-11521. 
Singh, H.; Srivastava, H. (2019): Jacobi collocation method for the approximate solution of some fractional-order Riccati differential equations with variable coefficients. Physica A: Statistical Mechanics and its Applications, vol. 523, pp. 1130-1149.

Sun, H.; Zhang, Y.; Baleanu, D.; Chen, W.; Chen, Y. (2018): A new collection of real world applications of fractional calculus in science and engineering. Communications in Nonlinear Science and Numerical Simulation, vol. 64, pp. 213-231.

Yüzbaşı, S. (2013): Numerical solutions of fractional Riccati type differential equations by means of the Bernstein polynomials. Applied Mathematics and Computation, vol. 219, no. 11 , pp. 6328-6343. 